\title{
The Effectiveness of Lunges and Static Stretching Exercises On Pain And Disability In Chronic Patellar Tendinopathy: A Case Report
}

\author{
Stasinopoulos Dimitrios \\ Department of Physiotherapy, University of West Attica, Greece
}

*Corresponding author: Stasinopoulos Dimitrios, Department of Physiotherapy, University of West Attica, Member of Laboratory of Neuromuscular \& Cardiovascular Study of Motion (LANECASM), Agiou Spyridonos 28, Egaleo 12243, Athens, Greece

\begin{abstract}
Eccentric exercises are not effective for all patients with chronic patellar tendinopathy (CPT). The aim of the present case report is to present the effect of eccentric - concentric loading combined with stretching exercises on pain and disability in a patient experiencing CPT. A patient with unilateral CPT for 7 months was included in the present report. The patient followed a supervised exercise program five times per week for 6 weeks consisting of slow progressive eccentric - concentric loading of quadriceps using forward step lunge (FSL) with the anterior knee motion going in front of the toes (FT) and static stretching exercises of hamstrings and quadriceps. The program was individualized on the basis of the patient's description of pain experienced during the procedure. The patient was evaluated using the VISA-P questionnaire at baseline, at the end of treatment (week 6), and 1 month (week 10) after the end of treatment. At the end of the treatment and at the follow-up there was a decline in pain and a rise in function. The results of the present trial suggest that the combination of slow progressive eccentric - concentric loading of quadriceps using forward step lunge (FSL) with the anterior knee motion going in front of the toes (FT) and static stretching exercises of hamstrings and quadriceps can produce significant improvements in terms of pain and disability in CPT.
\end{abstract}

Keywords: Patellar Tendinopathy; Lunges; Stretching; Jumper's Knee

\section{Introduction}

Chronic Patellar Tendinopathy (CPT) commonly referred to as Jumper's knee is the most common tendinopathy in the knee area. It is a degenerative condition and not an inflammatory one. Pain and decreased function are the main symptoms of CPT. Diagnosis is simple. The symptoms are reproduced by (1) lower limb activities such as squat or hop; (2) palpation on the site of pain (mainly at the inferior pole of the patella); and (3) clinical tests such as decline test [1]. No ideal treatment has emerged for the management of CPT. Many clinicians advocate a conservative approach [1] and physiotherapy is usually recommended [2]. A wide array of physiotherapy treatments has been recommended for the management of CPT such as electrotherapeutic/physical modalities, exercise program $\mathrm{s}$, soft tissue manipulation, and manual techniques [3]. These treatments have different theoretical mechanisms of action, but all have the same aim, to reduce pain and improve function. Such a variety of treatment options suggests that the optimal treatment strategy is not known, and more research is needed to discover the most effective treatment in patients with CPT. One of the most common physiotherapy treatments for CPT is exercise. Eccentric exercise has shown good clinical results in CPT [4] as well as in conditions similar to CPT in clinical behaviour and histopathological appearance, such as lateral elbow [5], rotator cuff [6] and Achilles tendinopathy [4]. Eccentric training is not enough for all patients with CPT [7]. Malliaras and his colleagues [8] concluded that clinicians should consider eccentricconcentric loading alongside or instead of eccentric loading in lower limb tendinopathy. Tightness of hamstring and quadriceps has been found in patients with CPT as an aetiological factor [9]. Thus, stretching exercises of the above muscles are needed in the rehabilitation program. To our knowledge, there have been no studies to investigate the effectiveness of eccentric-concentric loading and stretching exercises for the management of CPT. It is possible to combine eccentric-concentric loading with stretching exercises to see if the combination of these two therapeutic 
approaches offers results in the management of CPT. Therefore, the aim of the present case report is to present the effect of eccentricconcentric training combined with stretching exercises on pain and disability in a patient experiencing CPT.

\section{Case Presentation}

\section{History}

The subject was a 26-year-old female volleyball player with a seventh- month history of anterior knee pain, in her right knee. She was diagnosed by a specialist (orthopaedic) as having CPT. She has played volleyball for about 12 years. The site of pain was over the inferior pole of the patella without spreading down and she complained of pain during her training walking downstairs. The pain subsided within one or two hours after her training. She did not complain of pain after prolonged sitting. She did not have any problems with the other joints. She did not complain of other symptoms such as stiffness, swelling, locking, crepitus or giving away. She took no drugs at the time of assessment; she had no history of trauma in the knee before, only four ankle sprains in the other leg. She had followed a physiotherapy rehabilitation program following the ankle sprains. She had no prior physiotherapy treatment for the problem in her knee. She did not have a history of diabetes, epilepsy or cancer and none in her family did. She did not have any operation or illness in the past.

\section{Examination Findings}

Although the condition was diagnosed by a specialist, the physiotherapist D. S. assessed her knee to rule out other conditions and confirm the diagnosis. No pain was mentioned during gait and posture. Body deformity, colour changes, muscle wasting, or swelling were not noted. In palpation, signs of inflammatory activity like heat, swelling and synovial thickening were not found. On physical examination, the movements of the low back, hip and ankle were pain free, with full range of motion and full power. All ligamentous stress tests were normal, meniscal stress tests were normal, muscle strength tests were normal and no capsular pattern was found. Isotonic resisted extension reproduced mild pain on the inferior pole of the patella; what is more, after ten fast squats (decline test) [8], she experienced a mild pain. The squats were carried out, because the researcher wanted to reproduce the pain. Knee extension by gliding the patella medially was negative, without reproducing the pain; furthermore, the position of the patella was normal [10]. These two latter procedures ruled out the patellofemoral joint dysfunction. Tenderness with palpation over the inferior pole of the patella was found, confirming the diagnosis.

\section{Procedure}

The patient followed a supervised exercise program consisting of eccentric-concentric loading and static stretching exercises of hamstrings and quadriceps. As eccentric-concentric training, the participant carried out three sets of 15 repetitions of forward step lunge (FSL) with the anterior knee motion going in front of the toes (FT) with 1-min rest interval between each set. The FSLFT was performed at a slow speed at every treatment session. The patient counted to 6 during the FSL-FT. As the subject moved from the standing to the FSL-FT position, the quadriceps muscle and patellar tendon by inference were loaded eccentrically; followed by concentric loading, as the injured leg was used to get back to the start position. At the beginning the load consisted of the body weight and the participant was standing with all her body weight on the injured leg. The subject was told to go ahead with the exercise even if she experienced mild pain. However, she was told to stop the exercise if the pain became disabling. When the FSL-FT was pain-free the load was increased by holding hand weights. Static stretching exercises of quadriceps and hamstrings were performed as described by Dimitrios and his colleagues [9] before and after the eccentric - concentric loading. Each stretch lasted 30 seconds and there was a one minute rest between each stretch.

Supervised exercise program was given five times a week for 6 weeks and was individualized on the basis of the patient's description of pain experienced during the procedure. The patient was instructed to use her knee during the course of the study but to avoid activities that irritated pain such as jumping, hopping and running [7-11]. She was also told to refrain from taking antiinflammatory drugs throughout the course of the study. Patient compliance was monitored using a treatment diary. Communication and interaction (verbal and non-verbal) between the therapist and patient was kept to a minimum, and behaviors sometimes used by therapists to facilitate positive treatment outcomes were purposefully avoided. For example, patients were given no indication of the potentially beneficial effects of the treatments or any feedback on their performance in the pre-application and postapplication measurements [12]. Pain and function were measured in the present study. The patient was evaluated at the baseline (week 0), at the end of treatment (week 6) and at 1 month (week 10) after the end of treatment. The VISA-P questionnaire was used to monitor the pain and function of patients. The instrument is a simple questionnaire, consisted of eight questions that takes less than five minutes to complete and once patients are familiar with it they will be able to complete most of it themselves. It is a valid and reliable outcome measure for patients with patellar tendinopathy [13].

\section{Results}

Table 1: VISA-P score before each evaluation.

\begin{tabular}{|c|c|}
\hline & VISA - P \\
\hline WEEK 0 & 48 \\
\hline WEEK 6 & 77 \\
\hline WEEK 10 & 81 \\
\hline
\end{tabular}

VISA-P score was 48 at the initial evaluation. At the end of the treatment (week 6), there was a rise in VISA -P score of 29 units. At week 10, the VISA-P score was 81 (Table 1). 


\section{Discussion}

The present study examined the effect of FSL-FT with static stretching exercises of hamstrings and quadriceps in a patient experiencing CPT and its findings have demonstrated significant improvements in terms of pain and disability. The results obtained from this case report are novel; as to date, similar studies have not been conducted. Alfredson, et al. [14] first proposed the eccentric training of the injured tendon. It is the most commonly used conservative approach in the treatment of tendinopathy. Unilateral squat eccentric training of the patellar tendon was the most commonly used conservative approach in the treatment of CPT [15] when the problem is at the inferior pole of the patella; however, no studies have investigated the effectiveness of training on other sites of patellar tendinopathy. Studies determining the effectiveness of exercises at other sites of patellar tendinopathy are needed. Later, it was found that the unilateral squat eccentric training on a $25^{\circ}$ decline board applied more load on the tendon [9]. However, squat eccentric training of the patellar tendon alone, on decline board or not, was not effective for many patients with CPT. Malliaras and his colleagues [4] concluded that clinicians should consider eccentricconcentric loading alongside or instead of eccentric loading in Achilles and patellar tendinopathy. A Heavy Slow Resistance (HSR) program is recommended in the management of lower limb tendinopathy $[16,17]$. The HSR program was produced equivalent pain and function improvement (VISA) than the Alfredson eccentric program, but significantly better patient satisfaction at six months follow-up. In the Achilles tendon, eccentric and HSR have recently been shown to yield similar clinical outcomes (VISA and patient satisfaction) at 1 year follow up. Based on the above findings, the HSR program can be recommended as an alternative to the Alfredson eccentric program lower limb tendinopathy rehabilitation for young active people. Recently, isometric exercises have been recommended to reduce and manage tendon pain increasing the strength at the angle of contraction without producing inflammatory signs [8-18]. Five repetitions of 45-second isometric mid-range quadriceps exercise at $70 \%$ of maximal voluntary contraction have been shown to reduce patellar tendon pain for 45 minutes post exercise and this was also associated with a reduction in motor cortex inhibition of the quadriceps that was associated with patellar tendinopathy [18]. The dosage of isometric contractions is based on clinical experience [8-18] and their effect on pain in patients with CPT requires further study. The 'Spanish squat is- used as isometric contraction and is useful when there is limited or no access to gym equipment [8]. However, conflicting results have been reported in terms of immediate and short-term pain relief [19]. Definitive conclusions about the effectiveness of isometric exercise in tendinopathy are yet to be made [19]. A component lacking from evidence-based programs is adequate potential to alter load distribution on the lower limb kinetic chain and increase the risk of lower limb tendinopathy [1-20]. It is our belief that the improvement of lumbo-pelvic control can be achieved by performing simple exercises such as single leg bridging in supine and four - point prone bridging exercises. Future research is needed to confirm this suggestion. In addition, hip extensors weakness has been associated with patellar tendinopathy [21]. Exercises to strengthen these muscle groups should be considered in exercise protocols and patellar tendinopathy. However, hip extensors were not strengthened in the present case trial because the strength of hip muscles in the assessment was normal. Functional activities such as jumping, cutting and sprinting should also be included in lower limb tendinopathy rehabilitation programs among athletes, but have so far not been included in popular programs in the literature [21]. These activities were included in the present study. The athlete carried out these activities in the court under the supervision of the gymnast. There are different techniques for lunges, including variations in step length, walking or jumping lunges, or different trunk positions [22]. Keeping the knee behind the toes is a common cue during performing a proper form of lunges [22]. Research is needed to find out 1 ) which technique of lunges is the most effective and 2) if the lunge is more effective treatment approach than squat for CPT patients and 3) the load applied to the patellar tendon during the lunges. The load of exercises was increased according to the patients' symptoms otherwise the results are poor [23]. Furthermore, eccentric exercises were performed at a low speed in every treatment session because this allows tissue healing [24]. Ice was not recommended at the end of the treatment because research has shown that ice as a supplement to an eccentric exercise program offers no benefit to patients with tendinopathy [25]. Finally, the avoidance of painful activities is crucial for tendon healing, because training during the treatment period increases patients' symptoms and delays tendon healing [26].

Eccentric exercises appear to reduce the pain and improve function. The mechanism by which eccentric training achieves these outcomes remains uncertain, as there is a lack of good quality evidence relating to physiological effects. The clinical improvement of the HSR group was accompanied by increased collagen turnover. It is unknown if the isometric contractions can reverse the pathology of the tendinopathy and in this case the pathology of CPT. Although a home exercise program can be performed any time during the day without requiring supervision from a therapist, our clinical experience has shown that patients fail to comply with the regimen of home exercise programs [25]. Although many ways can be recommended to improve the compliance of patients with the home exercise program such as phone calls, exercise monitors and better self-management education, it is believed that this problem can be solved by the supervised exercise programs performed in a clinical setting under the supervision of a therapist. It is believed because our experience has shown that many patients stopped the home exercise program without giving an explanation, whereas patients completed the supervised program. One possible reason why they continue the supervised exercise program could be the cost. In the supervised exercise program, the patients visit the therapist more times than the home exercise program, and this is more expensive. A future study will combine the both types of exercise program $\mathrm{s}$ in order to maximize the compliance of the patients. Even though the positive effects of such an exercise 
program in CPT have been reported in the present report, its study design limits the generalization of these findings. Future welldesigned clinical trials are needed to confirm the positive results of this case study establishing the effectiveness of such an exercise program in the management of CPT. In addition, structural changes in the tendons related to the treatment interventions and the longterm effects ( 6 months or more after the end of treatment) of these treatments are needed to investigate. Further research is needed to establish the possible mechanism of action of this treatment approach, and the cost effectiveness of such treatment, because reduced cost is an important issue for the recommendation of any given treatment.

\section{Conclusions}

The exercise program, consisting of FSL-FT and static stretching exercises of hamstrings and quadriceps had reduced the pain and improved the function in a patient with CPT at the end of the treatment and at one month follow-up. Further well-designed trials are needed to confirm the results of the present case report.

\section{References}

1. Kountouris A, Cook J (2007) Rehabilitation of Achilles and patellar tendinopathies. Best Pract Res Clin Rheumatol 21: 295-316.

2. Peers KH, Lysens RJ (2005) Patellar tendinopathy in athletes: current diagnostic and therapeutic recommendations. Sports Med 35: 71-87.

3. Stasinopoulos D. Patellar (2014) Tendinopathy May not Be the Proper Term for Patients with Clinical Diagnosis of Patellar Tendon Disorder. Trauma Mon 19: e15301.

4. Malliaras P, Barton C, Reeves N, Langberg H (2013) Achilles and patellar tendinopathy loading programmes. a systematic review comparing clinical outcomes and identifying potential mechanisms for effectiveness. Sports Med 43: 267-286.

5. Raman J, MacDermid JC, Grewal R (2012) Effectiveness of different methods of resistance exercises in lateral epicondylosis-a systematic review. J Hand Ther 25: 5-25.

6. Camargo PR, Alburquerque-Sendín F, Salvini TF (2014) Eccentric training as a new approach for rotator cuff tendinopathy: Review and perspectives. World J Orthop 5: 634-644.

7. Cannell LJ, Taunton JE, Clement DB, Smith C, Khan KM (2001) A randomized clinical trial of the efficacy of drop squats or leg extension/ leg curl exercises to treat clinically diagnosed jumper's knee in athletes: pilot study. Br J Sports Med 35: 60-64.

8. Malliaras P, Cook J, Purdam C, Rio E (2015) Patellar Tendinopathy: Clinical Diagnosis, Load Management, and Advice for Challenging Case Presentations. J Orthop Sports Phys Ther 45: 887-898.

9. Dimitrios S, Pantelis M, Kalliopi S (2012) Comparing the effects of eccentric training with eccentric training and static stretching exercises in the treatment of patellar tendinopathy. A controlled clinical trial. Clin Rehabil 26: 423-430.

10. McConnell J (1989) The management of Chondromalacia Patellae. A long-term solution. Australian Journal of Physiotherapy 32: 24-32.
11. Stasinopoulos D (2015) The Effectiveness of Isometric Contractions Combined with Eccentric Contractions and Stretching Exercises on Pain and Disability in Lateral Elbow Tendinopathy. A case Report. J Novel Physiother 5: 1-5.

12. Vicenzino B, Collins D, Wright A (1996) The initial effects of a cervical spine manipulative physiotherapy treatment on the pain and dysfunction of lateral epicondylalgia. Pain 68: 69-74.

13. Visentini PJ, Khan KM, Cook JL, Kiss ZS, Harcourt PR, et al. (1998) The Visa score: An index of severity of symptoms in patients with jumper's knee (patellar tendinosis). Victorian institute of sport tendon study group. J Sci Med Sport 1: 22-28.

14. Alfredson H, Pietilä T, Jonsson P, Lorentzon R (1998) Heavy-load eccentric calf muscle training for the treatment of chronic Achilles' tendinosis. Am J Sports Med 26: 360-366.

15. Cook J, Karim M, Purdam C (2001) Conservative treatment of patellar tendinopathy. Phys Ther Sport 2: 54-65.

16. Beyer R, Kongsgaard M, Hougs Kjær B, Øhlenschlæger T, Kjær M et al. (2015) Heavy Slow Resistance Versus Eccentric Training as Treatment for Achilles Tendinopathy: A Randomized Controlled Trial. Am J Sports Med 43: 1704-1711.

17. Kongsgaard M, Kovanen V, Aagaard P, Doessing S, Hansen P, et al. (2009) Corticosteroid injections, eccentric decline squat training and heavy slow resistance training in patellar tendinopathy. Scand J Med Sci Sports 19: 790-802.

18. Rio E, Kidgell D, Purdam C, Gaida J, Moseley GL, et al. (2015) Isometric exercise induces analgesia and reduces inhibition in patellar tendinopathy. Br J Sports Med 49: 1277-1283.

19. Clifford C, Challoumas D, Paul L, Grant Syme, Neal L Millar, et al. (2020) Effectiveness of isometric exercise in the management of tendinopathy: a systematic review and meta-analysis of randomised trials. BMJ Open Sport \& Exercise Medicine 5: e000760.

20. Dimitrios S (2013) Achilles Tendinopathy and Lumbopelvic Stability. Rheumatology 4: 126.

21. Silva RS, Ferreira AL, Nakagawa TH, Santos JE, Serrão FV (2015) Rehabilitation of Patellar Tendinopathy Using Hip Extensors Strengthening and Landing Strategy Modification: Case Report With 6-Months Follow-Up. J Orthop Sports Phys Ther 21: 1-36.

22. Zellmer M, Kernozek TW, Gheidi N, Hove J, Torry M (2019) Patellar tendon stress between two variations of the forward step lunge. J Sport Health Sci 83: 235-241.

23. Jensen K, Di Fabio RP (1989) Evaluation of eccentric exercise in treatment of patellar tendinitis. Phys Ther 69: 211-216.

24. Kraushaar B, Nirschl R (1999) Current concepts review-tendinosis of the elbow (tennis elbow). Clinical features and findings of histological immunohistochemical and electron microscopy studies. J Bone Joint Surg Am 81: 259-285.

25. Manias P, Stasinopoulos D (2006) A controlled clinical pilot trial to study the effectiveness of ice as a supplement to the exercise programme for the management of lateral elbow tendinopathy. Br J Sports Med 40: 8185.

26. Visnes H, Hoksrud A, Cook J, Bahr R (2005) No effect of eccentric training on jumper's knee in volleyball players during the competitive season: a randomized clinical trial. Clin J Sport Med 15: 227-234. 


\section{(c) (i) This work is licensed under Creative}

To Submit Your Article Click Here: $\quad$ Submit Article

DOI: $10.32474 /$ OSMOAJ.2021.05.000207

$\begin{gathered}\text { Orthopedics and Sports Medicine } \\ \text { Open Access Journal }\end{gathered}$
Assets of Publishing with us
- Global archiving of articles
- Immediate, unrestricted online access
- Rigorous Peer Review Process
- Authors Retain Copyrights
Orthopedics And Sports Medicine:
Open Access Journal

